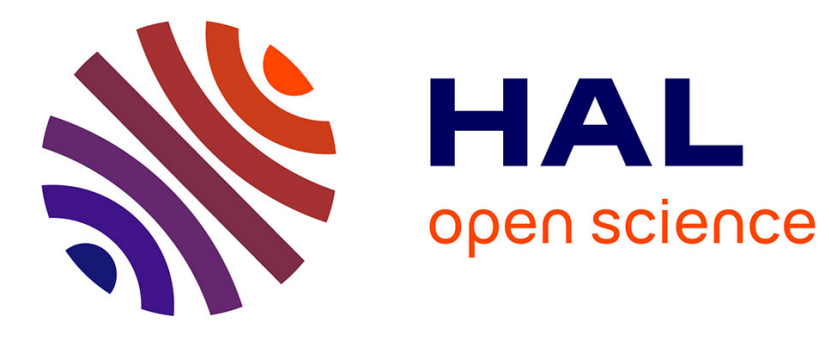

\title{
Selective Hardening Methodology for Combinational Logic
}

Samuel Nascimento Pagliarini, Lirida Alves de Barros Naviner, Jean-François Naviner

\section{- To cite this version:}

Samuel Nascimento Pagliarini, Lirida Alves de Barros Naviner, Jean-François Naviner. Selective Hardening Methodology for Combinational Logic. IEEE Latin-American Test Workshop (LATW), Apr 2012, Quito, Ecuador. pp.6. hal-00695808

\section{HAL Id: hal-00695808 https://hal-imt.archives-ouvertes.fr/hal-00695808}

Submitted on 9 May 2012

HAL is a multi-disciplinary open access archive for the deposit and dissemination of scientific research documents, whether they are published or not. The documents may come from teaching and research institutions in France or abroad, or from public or private research centers.
L'archive ouverte pluridisciplinaire HAL, est destinée au dépôt et à la diffusion de documents scientifiques de niveau recherche, publiés ou non, émanant des établissements d'enseignement et de recherche français ou étrangers, des laboratoires publics ou privés. 


\title{
Selective Hardening Methodology for Combinational Logic
}

\author{
Samuel N. Pagliarini, Lirida A. de B. Naviner and Jean-François Naviner \\ Institut TELECOM, TELECOM-ParisTech, LTCI-CNRS \\ 46, rue Barrault, 75634 - Paris Cedex 13, France \\ E-mail: \{samuel.pagliarini, lirida.naviner, jean-francois.naviner\}@ telecom-paristech.fr
}

\begin{abstract}
Defects as well as soft errors are a growing concern in micro and nanoelectronics. Multiple faults induced by single event effects are expected to be seen more often. Thus, reliability has become an important design criterion. In this context we introduce a cost-aware methodology for selective hardening of combinational logic cells. The methodology is based on the SPRA algorithm for calculating logical masking, and it is capable to automatically perform a trade-off between reliability improvements and associated costs, providing a list of the most effective candidates for hardening. The methodology is applied to a set of benchmark circuits using costs extracted from an actual standard cell library. The results then show that the methodology is able to diminish the unreliability of circuits in a cost-effective manner.
\end{abstract}

Index Terms-Reliability, Single Event Effects, Selective Hardening, Logic Masking.

\section{INTRODUCTION}

The amount of defects as well as the number of soft errors (i.e., transient errors) in electronic circuits are expected to increase, becoming a major concern in current and future technologies [1]. Thus, there is a current trend in the design of these circuits in which reliability related criterion are more and more common in the design flows.

Transient errors have many different sources. The transient faults that originate the errors can be caused by different physical phenomena, such as high-energy particle hits originating from cosmic rays, capacitive coupling, electromagnetic interference, or power transients [2]. Transient errors induced by the strikes of energetic particles at the devices are of great concern, specially for dependable systems and circuits $[1,3]$. In the past, transient errors used to be a concern only in the design of memories, thus resulting in the now widely used error correcting codes and other mitigation techniques such as nodal interleaving. On the other hand, at $90 \mathrm{~nm}$ and beyond, the reduced dimensions of the devices and also reduced operating voltage levels lead to more common radiation-induced faults in logic, with resulting error rates approaching those of memories $[4,5]$.

Therefore, transient errors in logic circuits are becoming an important reliability concern for current and future technology. Furthermore, the critical charge and the distance between sensitive junctions are decreasing with scaling. Thus, the energy of radiation particles that is required to cause multiple transient faults is also decreasing. The probability that a single high energetic particle affects the output of more than one circuit node, causing multiple faults, is no longer negligible [6].

On the ocasion of a transient fault, one or multiple electrical pulses may be created. These pulses, typically current spikes, are able to change the current output value of a node from one to zero or vice-versa. Yet, not all transients are able to reach the outputs of the combinational logic since there are natural masking properties against transient errors. These are:

- Electrical masking, which accounts for the attenuation of the current pulses as they propagate through the logic.

- Temporal masking, or latch-window masking, which defines a time interval in which the transients that reach the memory elements of the circuit will or will not be registered.

- Logical masking, which accounts for the lack of sensitized paths (from the erroneous node) to a primary output or memory element.

Among the masking phenomena that render immunity to combinational logic circuits from soft errors, logical masking is the hardest to model and characterize [7]. Also, logical masking is technology-independent. Thus, in this work we focus on calculating the reliability of a circuit considering only the logical masking. The other masking properties can be interpreted as derating factors, i.e., by calculating only the logical masking we are providing a underestimate of the actual circuit reliability. Electrical and latching-window masking computations may actually filter out some of the errors that logical masking does not filter.

Hardening techniques are applied in order to mitigate transient errors. Traditional hardening consumes too much area and/or energy to be cost-effective in commercial applications. Selective hardening, applied only to a design's most errorsensitive parts, offers an attractive alternative [8, 9]. Thus, considering logical masking, the main idea is to classify the composing blocks (i.e., standard cells) of a circuit according to their relative significance with respect to the reliability of the circuit. With the classified list of blocks it is possible to apply selective hardening either by using hardening by design techniques or by more generic fault tolerance techniques like Triple Modular Redundancy (TMR). By using an additional hardening affinity parameter, a trade-off between the hardening cost of a block and the reliability gain is then clearly stablished. 


\section{A. Related work}

In [9] we have studied the same problem of selective hardening but considering only single faults and by applying a different simulation/emulation model for obtaining the reliability figures. Such reliability methodology is referred as Probabilistic Binomial Reliability (PBR) [10]. The main drawback of the PBR methodology is the need for fault simulation or fault emulation, tasks that are inherently time consuming.

In [11] the authors have described a very similar approach for selective hardening. They have chosen an algorithm with linear complexity and accept the fact that innacurate values will be used for estimating the relative reliability gains of hardening a given cell in a circuit. We have used the same premise in our work. Yet, they are only concerned with single faults. Also, they propose a cost target such that the number of hardened cells does not exceed a cost limit $L$. All nodes have the same weight if this hypothesis is used while in our approach each type of node has a different cost. For instance, it is quite less costy to triplicate an inverter than to triplicate an or gate with several inputs.

\section{B. Contribution}

Our contribution is to provide an efficient methodology for selective hardening of the most critical blocks of a digital circuit. This methodology takes into account the effects of multiple faults since these are more prone to happen nowadays. Our methodology is also scalable since it relies in an algorithm with linear complexity to calculate the logical masking effect. A parameter similar to a hardening cost is also introduced, which allows the designer to drive the methodology using accurate cost values for the hardening of each block.

\section{Overview}

This paper is organized as follows: Section II contains the basic definitions used by our reliability estimation method. Section III describes the steps used to build the methodology to classify the blocks. It also contains a comparison among an accurate reliability estimation method and the approach we have used. Finnaly, in Section IV we have applied the proposed methodology in a series of circuits of different types, while in Section $\mathrm{V}$ we present our final remarks.

\section{PRELIMINARIES}

The reliability of a given circuit is the degree of confidence observed in the outputs of this circuit, given a certain scenario in which faults are expected to occur with a given probability. In this work we obtain the reliability figures of a circuit by applying the Signal Probability Reliability Analysis (SPRA) algorithm [12]. Based on a straightforward signal reliability computation and propagation algorithm, SPRA allows the evaluation of the logical masking capability of combinational logic circuits. Let us then define signal reliability.

\section{A. Signal reliability}

We consider the signal reliability of a given signal as the probability that this signal carries a correct value [12]. So, it is assumed that a binary signal $x$ can also carry incorrect information. This results in the fact that $x$ can take four different values: correct zero $\left(0_{c}\right)$, correct one $\left(1_{c}\right)$, incorrect zero $\left(0_{i}\right)$ and incorrect one $\left(1_{i}\right)$. Then, the probabilities for occurrence of each one of these four values are represented in matrices, as shown bellow:

$$
\left[\begin{array}{ll}
P\left(x=0_{c}\right) & P\left(x=1_{i}\right) \\
P\left(x=0_{i}\right) & P\left(x=1_{c}\right)
\end{array}\right]=\left[\begin{array}{ll}
x_{0} & x_{1} \\
x_{2} & x_{3}
\end{array}\right]
$$

The signal reliability for $x$, noted $R_{x}$, comes directly from expression (2), where $P($.$) stands for the probability function:$

$$
R_{x}=P\left(x=0_{c}\right)+P\left(x=1_{c}\right)=x_{0}+x_{3}
$$

\section{B. Reliability of a block}

Digital circuits are composed of many connected blocks, typically standard cells. Let us consider one of these blocks which performs a function on a signal $x$ in order to produce a signal $y$, i.e., $y$ is the output of the block. The probability that this block fails is given by $p$, such that $(0 \leq p \leq 1)$. Thus, $q=(1-p)$ is the probability it works properly. We can obtain the reliability of the signal $y$ as:

$$
R_{y}=\left(x_{0}+x_{3}\right) \cdot q+\left(x_{1}+x_{2}\right) \cdot p
$$

Equation (3) shows that, when the input signal is reliable, the output signal reliability is given by $q$. This implies that for fault-free inputs, the reliability of the output signal is given by the inherent reliability of the block that produces this signal. More complex scenarios are evaluated by also taking into account the truth table of the logic blocks.

SPRA uses both the reliability of the blocks as well as signal reliability to determine the cumulative effect of multiple simultaneous faults in the reliability of a circuit. To each signal in the circuit a signal reliability matrix is attributed. The propagation of these signals through the logic blocks is done by performing operations (tensoring and multiplying) in these matrices to calculate the actual reliability of the entire circuit. The basis for the SPRA algorithm comes from the Probabilistic Transfer Matrices algorithm (PTM) [13, 14]. The main difference is that the PTM approach does not scale well, while the SPRA approach has a linear complexity.

\section{Selective Hardening Methodology}

The reliability of a circuit consisting of several blocks depends on the reliabilities of these individual blocks. This is shown in equation (4) for a circuit consisting of $K$ blocks, where $R$ is the circuit's reliability and $q_{i}, q_{j}$ stand for the reliabilities of the blocks $b_{i}, b_{j}$ respectively $(1 \leq i, j \leq K)$.

$$
R=f\left(q_{1}, q_{2}, \ldots q_{i}, \ldots q_{j}, \ldots q_{K}\right)
$$


Assume that the blocks are independent in the sense that changing the reliability of a given block $b_{i}$ has no impact on the reliability of another block $b_{j}$ with $i \neq j$.

If we consider that a reliability change of a single block $b_{i}$ brings in its new reliability $q_{i}^{*}$, the circuit's reliability becomes $R_{i}^{*}$. Because different blocks $b_{i}$ and $b_{j}$ make different contributions to the reliability of a circuit, changes of different blocks may produce different values $R_{i}^{*}$ and $R_{j}^{*}$ [9].

In our methodology we assume that there is a hardening technique that is able to improve the reliability of a given block $b_{i}$, such that $q_{i}^{*}=1$. This is not a restriction, it is just a simplification, other values are also possible. Then, for all blocks of the circuit we perform an evaluation run of the SPRA algorithm. In each evaluation run we select a node $b_{i}$, allow $q_{i}^{*}$ to be 1 , and obtain the new reliability value $R_{i}^{*}$. This effort is possible since the complexity of the SPRA algorithm is linear.

After all initial evaluation runs are performed, we obtain a list of all $R_{i}^{*}$ values. At this point, one could sort the list and elect the block with the highest $R_{i}^{*}$ to be hardened. This is the approach followed in [9]. Yet, we are interested in stablishing a trade-off between the cost of hardening this block against the cost of hardening any other block. In order to do so, we introduce a new parameter to express the hardening affinity of such block, given by $H a_{i}$.

The parameter $H a_{i}$ of each type of cell is defined by the user. It must be constrained in the interval $[0,1]$. This parameter is generic and can be used to express any type of hardening trade-off: area, delay, power or combinations of the previous.

It can also be used to model other situation of particular interest: assume that a certain cell is available in two versions in the same standard cell library, let us say $I N V_{t}$ and $I N V_{h}$. The former is a traditional design while the latter is a hardened by design version of an inverter. Assume that the hardening by design, once is already done, has no additional cost to be implemented. Thus, to model this situation one should say that this particular cell has the highest hardening affinity, i.e., the $H a_{i}$ of $I N V_{h}$ is 1 . Since the other types of cells have no hardened versions available, they will require a technique such as TMR to be applied. This technique is expected to have a larger cost and therefore any cell requiring TMR will have a $H a_{i}<1$.

In Tab. I we show the values that were used in our experiments for some cells. These values are extracted from an actual 90nm standard cell library provided by Synopsys [15]. In our analysis we considered that only the dynamic power of the blocks would be considered to calculate the hardening affinity. So, for each cell in the library, we have divided the dynamic power of the smallest inverter in the library by the given cell actual dynamic power. It is possible to notice that negated cells (like NOR and NAND) benefit from the CMOS natural inversion and have a higher hardening affinity. It is also possible to notice that inverters have the smallest dynamic power of all cells. All the other $H a_{i}$ values are normalized.

After each cell's affinity is known it is necessary to apply
TABLE I: Hardware affinity $\left(H a_{i}\right)$ parameters for several blocks.

\begin{tabular}{c|r|r}
\hline Block & Power $(\mathrm{nW} / \mathrm{MHz})$ & Hardening affinity \\
\hline INVX0 & 10 & 1 \\
NAND2X0 & 3583 & 0.002790957 \\
NOR2X0 & 4211 & 0.002374733 \\
AND2X1 & 6545 & 0.001527884 \\
OR2X1 & 6859 & 0.001457938 \\
OR4X1 & 7698 & 0.001299039 \\
MUX21X1 & 8639 & 0.001157541 \\
XOR2X1 & 8702 & 0.001149161 \\
AOI21X1 & 13912 & 0.000718804 \\
\hline
\end{tabular}

this value to decide wich block should be selected for hardening. This step of the methodology introduces a new value, the reliability gain or reliability difference, given by $R g_{i}$. This is the difference from the circuit reliability before and after a single block was hardened. For each evaluation run this value is calculated as follows:

$$
R g_{i}=R_{i}^{*}-R
$$

The value of (5) is then used to calculate the reliabilityaffinity product as follows:

$$
\operatorname{Prh}_{i}=R g_{i}^{w 1} \times H a_{i}^{w 2}
$$

where $w 1$ and $w 2$ are the weigths to be applied. I.e., the user may choose if reliability should be more important than power or vice-versa, and by which amount. In the experiments that are presented in Section IV, these values were set as $w 1=2$ and $w 2=1$.

Once the value of (6) has been calculated for all cells, these are sorted and the highest value is picked. This block is then assumed to be hardened and the new circuit reliability $\left(R_{i}^{*}\right)$ is obtained. This reliability is then compared against a user-given reliability target. If it is lower than the target the methodology algorithm starts again and all cells still not hardened are considered as candidates. Otherwise, if the target is met, the algorithm ends and outputs the ordered lists of cells to be hardened.

\section{A. Comparison with an accurate reliability analysis algorithm}

Our methodology uses the SPRA algorithm which is not accurate. The sources of inaccuracies come from incorrect evaluation of multiple fanout branches. An accurate analysis is possible using the multi-pass algorithm described in [12], here referred as SPRMP. It is well known that both algorithms produce different values for the reliability of a circuit. Yet, we are interested in comparing how well SPRA estimates the critical node in comparison with SPRMP.

Let us first consider a simple circuit, $c 17$, which has only 6 nodes. By applying both algorithms just once, i.e., neglecting a reliability target given by the user, two lists of $b_{i}$ nodes are 
created. These lists are sorted according to the $R_{i}^{*}$ of each node and are referred as $L_{b}$. These lists are showed in Tab. II.

The meaning of each column of Tab. II is as follows:

- Position is the ranking of the nodes according to $R_{i}^{*}$.

- SPRMP's $L_{b}$ is the list of nodes generated by the SPRMP algorithm, i.e., the accurate list.

- SPRA's $L_{b}$ is the list of nodes generated by the SPRA algorithm, i.e., the inaccurate list.

- Position difference is the difference in the ranking from column 3 with respect to column 2. E.g., block 4 is ranked first in the SPRA's $L_{b}$ list while it is ranked second in the SPRMP's $L_{b}$ list, thus the difference is 1 position.

- Normalized difference is the position difference divided by the maximum position error possible. In this example it is 5 since the circuit has 6 blocks.

According to the analysis of the circuit $c 17$ presented in Tab. II, the average error introduced by the SPRA algorithm is $13.3 \%$. Yet, we have performed this same analysis for other circuits with different profiles, all containing multiple fanouts branches. The selected circuits are very limited in size since the execution time of the SPRMP algorithm is very high even for medium-sized circuits. The details of the chosen circuits are as follows:

- 74283, a 4 bit adder.

- AOI, which contains an and-or-inverter logic and 2 multiple fanouts.

- AOIX2, which contains a larger and-or-inverter logic followed by a buffer network with many multiple fanouts.

- decoder, which contains a large or-like logic to decode 8 inputs. Each input feeds many gates so multiple fanouts appear already in the inputs.

- chain, which contains a chain of inverters and or gates.

Table III summarizes the comparison of all the presented circuits plus the already presented $c 17$ one.

The results in Tab. III clearly show that for some circuits both algorithms produce the same list of blocks to be hardened. Yet, for the circuits $c 17,74283$ and AOIX2 the maximum error is quite high. A deeper analysis is performed for such circuits, where the error distribution is analysed.

The error distribution for the 74283 circuit is shown in Fig. 1, where the nodes in the $\mathbf{x}$ axis are sorted according to $R_{i}^{*}$ (the actual labels are the ids of the blocks in the circuit).

TABLE II: Comparison of the SPRA and SPRMP algorithms.

\begin{tabular}{c|r|r|r|r}
\hline Position & SPRMP's $L_{b}$ & SPRA's $L_{b}$ & $\begin{array}{c}\text { Position } \\
\text { difference }\end{array}$ & $\begin{array}{c}\text { Normalized } \\
\text { difference }\end{array}$ \\
\hline 1 & 2 & 4 & 1 & 0.2 \\
2 & 4 & 5 & 1 & 0.2 \\
3 & 5 & 2 & 2 & 0.4 \\
4 & 1 & 1 & 0 & 0 \\
5 & 0 & 0 & 0 & 0 \\
6 & 3 & 3 & 0 & 0 \\
\hline \multicolumn{2}{r|}{ Average error: } & 0.133 \\
\hline
\end{tabular}

TABLE III: Comparison of the methodology using the SPRA and SPRMP algorithms.

\begin{tabular}{c|r|r|r}
\hline Circuit & Minimum error & Average error & Maximum error \\
\hline c17 & 0 & 0.133 & 0.4 \\
74283 & 0 & 0.07 & 0.28 \\
AOI & 0 & 0 & 0 \\
AOIX2 & 0 & 0.35 & 0.85 \\
decoder & 0 & 0 & 0 \\
chain & 0 & 0 & 0 \\
\hline
\end{tabular}

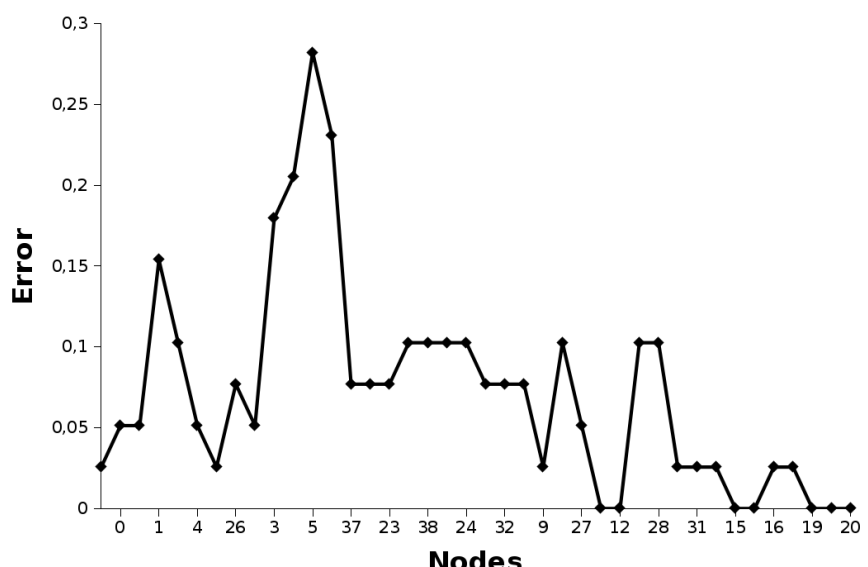

Fig. 1: Error distribution for the circuit 74283.

It is possible to notice that some blocks have a higher error probability. Yet, that is not the case for the blocks that are closer to the $\mathbf{y}$ axis (which are exactly the best candidates for hardening). This same profile, where the error is not that high in the elected block, is also seen in the error distribution of the other circuits. The same profile is also observed after some cells have already been elected for hardening. Thus, our results are presented using the SPRA algorithm only.

In the particular case of the AOIX2 circuit, the higher error values are due to the large number of multiple fanouts in the buffer network. Regarding the circuit $c 17$, it only has 6 cells. So any small difference is very meaningful, even in relative terms.

\section{EXPERIMENTAL RESULTS}

The methodology described in Section III was applied to several ISCAS benchmark circuits [16]. Each block from each circuit was set using $q_{i}=0.9999$. The reliability target was adjusted so a decrease of the unreliability would be reached for each circuit. The results are presented in tables IV and $\mathrm{V}$. The former table contains the results for a reduction of at least $20 \%$ (w.r.t. the original unreliability) while the latter contains the results for a reduction of at least $40 \%$. The original unreliability of each circuit is given in the second column of the aforementioned tables.

The column entitled "Original Power" contains the sum of the dynamic power of all cells of each circuit. The columns entitled "Hardened Cells" contain the amount of cells that 
TABLE IV: Results for decreasing the unreliability by at least $20 \%$.

\begin{tabular}{c|r|r|r|r|r|r}
\hline \multirow{2}{*}{ Circuit } & \multirow{2}{*}{$\begin{array}{c}\text { Original } \\
\text { Unreliability }\end{array}$} & $\begin{array}{c}\text { Original Power } \\
(\mathrm{nW} / \mathrm{MHz})\end{array}$ & \multicolumn{2}{|c|}{ No hardening affinity } & \multicolumn{2}{c}{ With hardening affinity } \\
\cline { 4 - 7 } & & & $\begin{array}{c}\text { Hardened } \\
\text { cells }\end{array}$ & Power $(\mathrm{nW} / \mathrm{MHz})$ & $\begin{array}{c}\text { Hardened } \\
\text { cells }\end{array}$ & Power $(\mathrm{nW} / \mathrm{MHz})$ \\
\hline$c 17$ & 0.000562 & 21498 & 1 & 21498 & 1 & 21498 \\
74283 & 0.003848 & 189244 & 4 & 222932 & 8 & $\mathbf{1 8 9 4 0 4}$ \\
$c 432$ & 0.013466 & 624686 & 9 & 624866 & 9 & 624866 \\
$c 499$ & 0.013611 & 1321460 & 20 & 1669540 & 41 & $\mathbf{1 3 2 2 2 8 0}$ \\
$c 1355$ & 0.021905 & 1907300 & 38 & 2179608 & 38 & 2179608 \\
$c 1908$ & 0.031668 & 2146539 & 58 & 2147699 & 58 & 2147699 \\
$c 3540$ & 0.062635 & $5.90419 \mathrm{e}+06$ & 54 & $5.90527 \mathrm{e}+06$ & 54 & $5.90527 \mathrm{e}+06$ \\
$c 2670$ & 0.064015 & $4.07731 \mathrm{e}+06$ & 41 & $4.12503 \mathrm{e}+06$ & 42 & $\mathbf{4 . 0 8 0 4 4 e + 0 6}$ \\
$c 5315$ & 0.085614 & $8.89708 \mathrm{e}+06$ & 59 & $8.96576 \mathrm{e}+06$ & 60 & $\mathbf{8 . 9 0 0 5 7 e + 0 6}$ \\
\hline
\end{tabular}

TABLE V: Results for decreasing the unreliability by at least $40 \%$.

\begin{tabular}{c|r|r|r|r|r|r}
\hline \multirow{2}{*}{ Circuit } & \multirow{2}{*}{$\begin{array}{c}\text { Original } \\
\text { Unreliability }\end{array}$} & \multirow{2}{*}{$\begin{array}{c}\text { Original Power } \\
(\mathrm{nW} / \mathrm{MHz})\end{array}$} & \multicolumn{2}{|c|}{ No hardening affinity } & \multicolumn{2}{c}{ With hardening affinity } \\
\cline { 4 - 7 } & & & $\begin{array}{c}\text { Hardened } \\
\text { cells }\end{array}$ & Power $(\mathrm{nW} / \mathrm{MHz})$ & $\begin{array}{c}\text { Hardened } \\
\text { cells }\end{array}$ & Power $(\mathrm{nW} / \mathrm{MHz})$ \\
\hline$c 17$ & 0.000562 & 21498 & 2 & 35830 & 2 & 35830 \\
74283 & 0.003848 & 189244 & 10 & 273464 & 16 & $\mathbf{1 8 9 5 6 4}$ \\
$c 432$ & 0.013466 & 624686 & 26 & 625206 & 26 & 625206 \\
$c 499$ & 0.013611 & $1.32146 \mathrm{e}+06$ & 48 & $2.15685 \mathrm{e}+06$ & 80 & $\mathbf{1 . 4 2 7 3 6 e + 0 6}$ \\
$c 1355$ & 0.021905 & $1.90730 \mathrm{e}+06$ & 83 & $2.50208 \mathrm{e}+06$ & 83 & $2.50208 \mathrm{e}+06$ \\
$c 1908$ & 0.031668 & $2.14653 \mathrm{e}+06$ & 132 & $2.14918 \mathrm{e}+06$ & 132 & $2.14918 \mathrm{e}+06$ \\
$c 3540$ & 0.062635 & $5.90419 \mathrm{e}+06$ & 175 & $5.90769 \mathrm{e}+06$ & 175 & $5.90769 \mathrm{e}+06$ \\
$c 2670$ & 0.064015 & $4.07731 \mathrm{e}+06$ & 128 & $4.22630 \mathrm{e}+06$ & 128 & $\mathbf{4 . 0 8 4 4 4 e + 0 6}$ \\
$c 5315$ & 0.085614 & $8.89708 \mathrm{e}+06$ & 205 & $9.13570 \mathrm{e}+06$ & 207 & $\mathbf{8 . 9 0 4 6 5 e + 0 6}$ \\
\hline
\end{tabular}

are elected for hardening. By using the hardening affinity parameter this number tends to increase. Then, the columns entitled "Power" contain the sum of the dynamic power of all cells of the new version of the circuit. A fairly simple assumption was made: on hardening a given node we should add three times the value of the power of that node to the overall circuit power.

Thus the additional power that would be consumed by a voter is not considered. Once again, this is a simplification. A voter should be considered for a group of cells and not for a single cell, otherwise the costs can become unfeasible. Assuming one voter for each hardened cell would create a large cost both in terms of area and power. Therefore the power figures given in the tables are a minimum value estimate. Voter placement (i.e., TMR granularity) is not the scope of this work.

In tables IV and V some power figures are highlighted in bold. It is clear that applying the methodology considering the hardening affinity is an effective trade-off between power and reliability in these cases. This does not mean that the methodology is not appropriate for the other circuits. In fact, it means that the current choice of parameters $w 1$ and $w 2$ is not a good one for the circuits that are not highlighted.

\section{A. Comparison}

A straightforward comparison with other methodologies is not simple since the goals are different. The results presented in [9] are in alignment with the results presented in this work, which is a strong suggestion that multiple faults do not have a large impact on the decision of which node to harden. Yet, they have a considerable effect on the actual reliability of the circuit. Thus, they are important when determining the tradeoffs between cost and reliability.

A radiation hardening technique for combinational logic is proposed in [17]. The hardening is achieved by increasing the gate size of some critical nodes in the circuit but no hardening against defects is mentioned. Thus the technique presented here is more of a general solution since it is technologyindependent. The overheads mentioned in [17] are not directly comparable.

Nevertheless, in qualitative terms it is easily observed that certain cells have a larger impact in the reliability of the circuit than others. This observation is highlighted in $[9,11,17]$. In our exeperiments this was also observed. There are some particular cases, like the one illustrated in Fig. 2, where choosing the correct node to harden has a large impact in the overall circuit reliability. The analysis depicted in Fig. 2 is from the circuit $c 1355$.

Regarding Fig. 2, it contains the $R_{i}^{*}$ values related to the hardening of all possible cells. The nodes in the $\mathbf{x}$ axis are ordered by the reliability gain that hardening that node would produce. The circuit was evaluated given the parameter $q_{i}=$ 0.9999 for each cell. In absolute terms the difference from the 


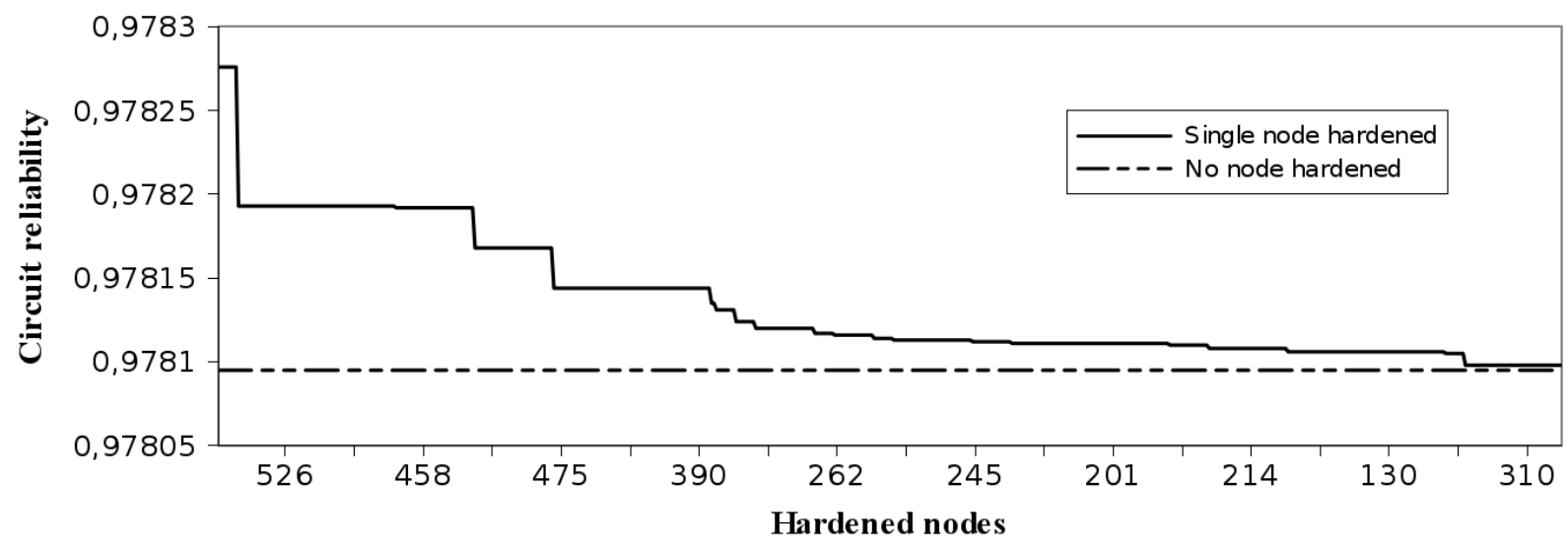

Fig. 2: Reliability gain versus chosen node to be hardened.

best to the worst candidate is not large. Yet, usually several cells are selected for hardening (as in Tab. V), so these values accumulate. Thus choosing the best candidate for hardening is critical.

\section{CONCLUSION}

In a context where defects and soft errors are a growing concern, we have proposed a selective hardening methodology for combinational logic nodes. Furthermore, we have also concerned multiple faults to determine the actual reliability of the circuits.

Our results present the use of the methodology in conjunction with a standard cell library from an actual vendor, where the trade-off between power and reliability gain is highlighted. Thus, the methodology can be integrated in commercial design flows in a very straightforward manner. Other cost-effective schemes are also possible since the methodology contains a generic parameter to define the hardening affinity of a node.

In our future works we plan to investigate the effects of multiple faults in the reliability of a circuit by limiting the effect of multiple faults locally in the circuit. The study of the relationship between parameters $w 1$ and $w 2$ is also a matter for a future work.

\section{ACKNOWLEDGMENT}

This work was partially funded by the CATRENE project RELY.

\section{REFERENCES}

[1] M. Nicolaidis, "Design for soft error mitigation," Device and Materials Reliability, IEEE Transactions on, vol. 5, no. 3, pp. 405 - 418, sept. 2005.

[2] G. Saggese, N. Wang, Z. Kalbarczyk, S. Patel, and R. Iyer, "An experimental study of soft errors in microprocessors," Micro, IEEE, vol. 25 , no. 6 , pp. 30 - 39, nov.-dec. 2005.

[3] P. Dodd and L. Massengill, "Basic mechanisms and modeling of single-event upset in digital microelectronics," Nuclear Science, IEEE Transactions on, vol. 50, no. 3, pp. 583 - 602, june 2003.

[4] P. Shivakumar, M. Kistler, S. Keckler, D. Burger, and L. Alvisi, "Modeling the effect of technology trends on the soft error rate of combinational logic," in Dependable Systems and Networks, 2002. DSN 2002. Proceedings. International Conference on, 2002, pp. 389 - 398.
[5] B. Narasimham, B. Bhuva, R. Schrimpf, L. Massengill, M. Gadlage, O. Amusan, W. Holman, A. Witulski, W. Robinson, J. Black, J. Benedetto, and P. Eaton, "Characterization of digital single event transient pulse-widths in 130-nm and 90-nm cmos technologies," Nuclear Science, IEEE Transactions on, vol. 54, no. 6, pp. $2506-2511$, dec. 2007.

[6] D. Rossi, M. Omana, F. Toma, and C. Metra, "Multiple transient faults in logic: an issue for next generation ics?" in Defect and Fault Tolerance in VLSI Systems, 2005. DFT 2005. 20th IEEE International Symposium on, oct. 2005 , pp. $352-360$.

[7] N. George and J. Lach, "Characterization of logical masking and error propagation in combinational circuits and effects on system vulnerability," in Dependable Systems Networks (DSN), 2011 IEEE/IFIP 41st International Conference on, june 2011, pp. $323-334$.

[8] I. Polian and J. Hayes, "Selective hardening: Toward cost-effective error tolerance," Design Test of Computers, IEEE, vol. 28, no. 3, pp. $54-63$, may-june 2011.

[9] L. A. de B. Naviner, J.-F. Naviner, T. Ban, and G. S. Gutemberg, "Reliability analysis based on significance," in Argentine School of Micro-Nanoelectronics Technology and Applications (EAMTA), 2011, aug. 2011, pp. $1-7$.

[10] M. de Vasconcelos, D. Franco, L. de B. Naviner, and J.-F. Naviner, "Reliability analysis of combinational circuits based on a probabilistic binomial model," in Circuits and Systems and TAISA Conference, 2008. NEWCAS-TAISA 2008. 2008 Joint 6th International IEEE Northeast Workshop on, june 2008, pp. $310-313$.

[11] I. Polian, S. Reddy, and B. Becker, "Scalable calculation of logical masking effects for selective hardening against soft errors," in Symposium on VLSI, 2008. ISVLSI '08. IEEE Computer Society Annual, april 2008, pp. $257-262$.

[12] D. T. Franco, M. C. Vasconcelos, L. Naviner, and J.-F. Naviner, "Signal probability for reliability evaluation of logic circuits," Microelectronics Reliability, vol. 48, no. 8-9, pp. 1586 - 1591, 2008

[13] K. N. Patel, I. L. Markov, and J. P. Hayes, "Evaluating circuit reliability under probabilistic gate-level fault models," in In International Workshop on Logic Synthesis (IWLS, 2003, pp. 59-64.

[14] S. Krishnaswamy, G. Viamontes, I. Markov, and J. Hayes, "Accurate reliability evaluation and enhancement via probabilistic transfer matrices," in Design, Automation and Test in Europe, 2005. Proceedings, march 2005 , pp. 282 - 287 Vol. 1.

[15] Synopsys Armenia Educational Department, "Saed 90nm generic library." [Online]. Available: http://www.synopsys.com/Community/ UniversityProgram

[16] F. Brglez and H. Fujiwara, "A neutral netlist of 10 combinational benchmark circuits and a target translator in fortran," in Proceedings of the International Symposium on Circuits and Systems, June 1985, pp. 663-698.

[17] Q. Zhou and K. Mohanram, "Cost-effective radiation hardening technique for combinational logic," in Computer Aided Design, 2004. ICCAD-2004. IEEE/ACM International Conference on, nov. 2004, pp. $100-106$. 